\title{
Discovery of two new methanol masers in NGC 7538
}

\section{Locating of massive protostars}

\author{
M. R. Pestalozzi ${ }^{1}$, V. Minier ${ }^{2,3}$, F. Motte ${ }^{3}$, and J. E. Conway ${ }^{4}$ \\ 1 School of Physics, Astronomy and Mathematics, University of Hertfordshire, AL10 9BS Hatfield, UK \\ e-mail: michele@star.herts.ac.uk \\ 2 Service d'Astrophysique, DAPNIA/DSM/CEA Saclay, 91191 Gif-sur-Yvette, France \\ 3 AIM, UMR 7158, CEA-CNRS-Université Paris VII, CEA/Saclay, 91191 Gif sur Yvette, France \\ 4 Onsala Space Observatory, 43992 Onsala, Sweden
}

Received 12 October 2005 / Accepted 19 January 2006

\section{ABSTRACT}

Context. NGC 7538 is known to host a 6.7 and $12.2 \mathrm{GHz}$ methanol maser cospatial with a Ultra Compact (UC) H II region, IRS 1.

Aims. We report on the serendipitous discovery of two additional $6.7 \mathrm{GHz}$ methanol masers in the same region, not associated with IRS 1.

Methods. Interferometry maser positions are compared with recent single-dish and interferometry continuum observations.

Results. The positions of the masers agree to high accuracy with the $1.2 \mathrm{~mm}$ continuum peak emission in NGC 7538 IRS 9 and NGC 7538 S. This clear association is also confirmed by the positional agreement of the masers with existing high resolution continuum observations at $\mathrm{cm}$ and/or mm wavelengths.

Conclusions. Making use of the established strong relation between methanol masers and high-mas star formation, we claim that we have accurately positioned the high-mass protostars within the regions where they are detected. The variety of objects hosting a $6.7 \mathrm{GHz}$ methanol maser in NGC 7538 shows that this emission probably traces different evolutionary stages within the protostellar phase.

Key words. star formation - massive stars - interstellar medium - masers

\section{Introduction}

The NGC 7538 nebula is a furnace of intense massive star formation located at some $2.7 \mathrm{kpc}$ from the Sun. At least 11 infrared sources have been identified embedded within it (Fig. 1, Ojha et al. 2004 and references therein). These IR sources lie in four regions hosting a large range of different types of young stellar objects (YSOs) with signs of decreasing age moving from the North-West to the South-East. In the far North-West the first star formation region contains the IR sources IRS 5, 6,7 which are associated with a developed $\mathrm{H}$ II region of $\sim 3 \mathrm{pc}$ in size. IRS 6 has been proposed as its exciting source (Ojha et al. 2004). A further star formation region can be identified with the IR sources IRS 1-3, where infrared emission at $K$-band and long-ward is dominated by IRS 1 (De Buizer \& Minier 2005). IRS 1 is known to power a Ultra Compact (UC) H II region, an early stage of massive star formation. Masers of different species have also been detected toward this source $(\mathrm{OH}$, $\mathrm{H}_{2} \mathrm{O}, \mathrm{CH}_{3} \mathrm{OH}$, see Minier et al. 1998 and references therein). A third star-forming region is centered on NGC $7538 \mathrm{~S}$ and also contains IRS $11,10^{\prime \prime}$ to its northwest (Fig. 1). Coincident with $\mathrm{NGC} 7538 \mathrm{~S} \mathrm{OH}$ and $\mathrm{H}_{2} \mathrm{O}$ masers have been detected (Kameya et al. 1990). Finally, furthest to the South-East, the fourth known region of star formation contains the deeply embedded IR source IRS 9, believed to be in a stage prior to the formation of a UC $\mathrm{H}$ II region. $\mathrm{H}_{2} \mathrm{O}$ masers were also detected toward this source (Davis et al. 1998; Kameya et al. 1990).

Methanol masers are indicative of the earliest stages of massive star formation. They arise from cold, embedded molecular clumps and in some cases from dark infrared clouds (Hill et al. 2005; Purcell et al. 2005). These clumps are often interpreted to be protoclusters of high mass YSOs, i.e. precursors of OB associations (e.g. Motte et al. 2003; Minier et al. 2005). High angular resolution observations of methanol maser environments reveal that these masers are associated with hot cores, UC H II regions and very often do not coincide with radio continuum emission from bright Ultra Compact (UC) H II regions (Walsh et al. 1998; Minier et al. 2001). The detection of a methanol maser in such regions is a clear indication of the presence of a deeply embedded hot core that is characterised by large methanol abundance and 100-200 K gas and dust temperature. These physical conditions are in agreement with those derived from maser modelling, as e.g. Sobolev et al. 1997. The high astrometric accuracy of VLBI observations of methanol masers gives thus the best estimate of the position of the high-mass protostellar core at a scale of $\sim 10 \mathrm{AU}$. 

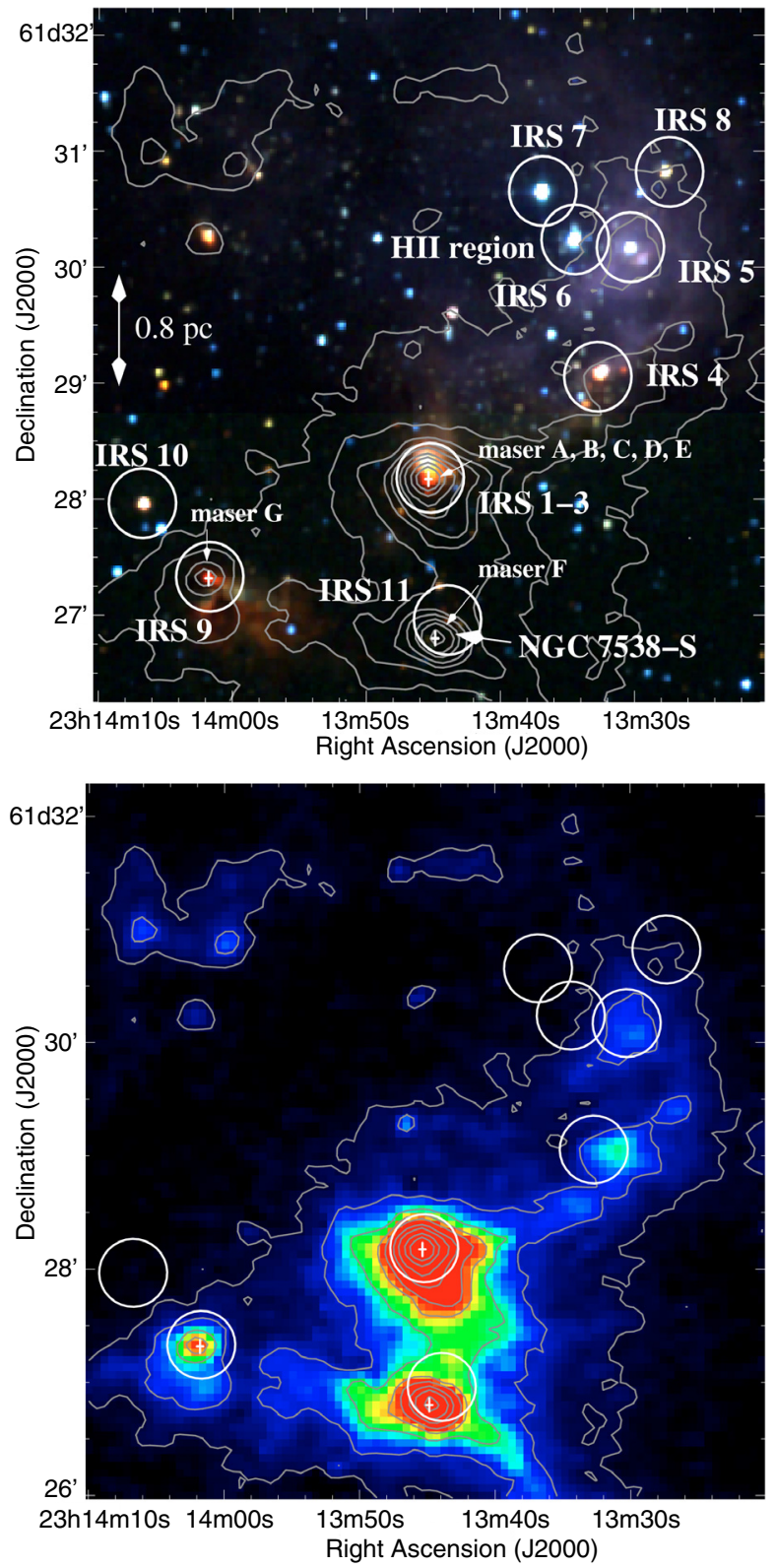

Fig. 1. General view of the NGC 7538 region. Left: the underlying colour image is a RGB image of $J, H, K$ NIR bands made from the 2MASS archive. The grey contours $(2,5,10,15,20,30,40,50,60$, $70,80,90 \%$ of peak emission, $\sim 4 \mathrm{Jy} \mathrm{beam}^{-1}$ ) show $1.2 \mathrm{~mm}$ dust continuum mapped with MAMBO-2/IRAM-30m. White circles indicate the location of the identified infrared sources. White crosses indicate the $6.7 \mathrm{GHz} \mathrm{CH}_{3} \mathrm{OH}$ maser positions. Right: the underlying image is the $1.2 \mathrm{~mm}$ continuum map. $1 \mathrm{arcmin}$ is $0.78 \mathrm{pc}$.

One clear example of this fact is given by the main component of the $\mathrm{CH}_{3} \mathrm{OH}$ maser in NGC 7538 (component $\mathrm{A}$ in Fig. 2): the maser pinpoints the massive protostellar core within the IRS 1 submm clump to milliarcsecond accuracy (1 mas at $\sim 3 \mathrm{kpc}$ is $\sim 3 \mathrm{AU}$, see Pestalozzi et al. 2004).

In this letter we report on the serendipitous detection of 6.7 GHz methanol masers in the third and fourth star formation regions in NGC 7538, cospatial with NGC7538 S and IRS 9. Supported by what exposed above, we argue that with this discovery we have accurately positioned the two massive

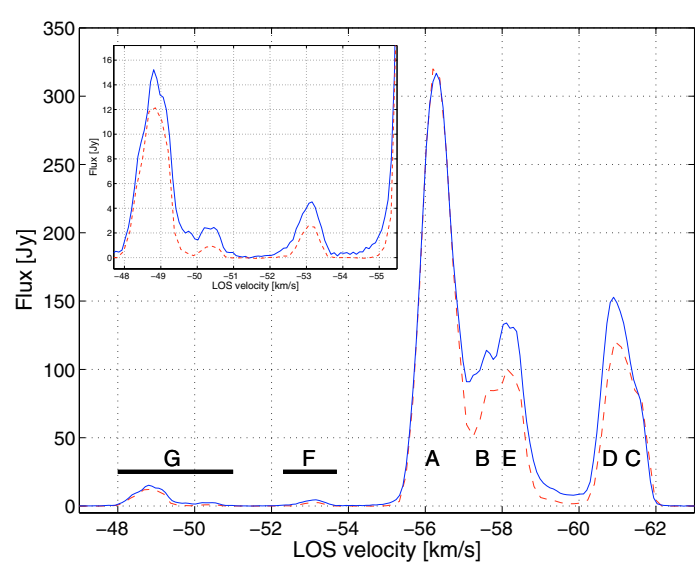

Fig. 2. Cross correlated spectrum on the KN-DA baseline averaged over the whole observing run (dashed line) and autocorrelation spectrum from the DA antenna (solid line), both from run1. Minimal fringe spacing on the KN-DA baseline is $\sim 190$ mas. Features A, B, C, Dand Eare located within 0.5 arcsec of IRS 1. The inset is a zoom-in of the velocity region including features $F \& G$. The multiplicity of $F \& G$ is discussed in the text.

protostars in those regions. This is then confirmed by the coincidence of the new masers with the peak of the $1.2 \mathrm{~mm}$ dust emission coming from the thick cocoon surrounding them.

\section{Observations and data reduction}

The data used for this paper was taken in two runs, with the principal aim of studying the known $6.7 \mathrm{GHz}$ methanol maser emission toward NGC 7538 (i.e. spectral components A-E, see Fig. 2). As explained below, the results presented here refer to the serendipitous discovery of two new maser features, unseen until now in any interferometry experiment.

The first data set (run1, November 4th-5th 2004) was obtained during a joint experiment including the European VLBI Network $^{1}$ and the three antennas of the MERLIN ${ }^{2}$ array which were then operational at $5 \mathrm{~cm}$ wavelength, Cambridge (CM), Knockin (KN) and Darnhall (DA). The spectral resolution was 1.9 and $3.9 \mathrm{kHz} / \mathrm{chan}$ (or 0.087 and $0.175 \mathrm{~km} \mathrm{~s}^{-1}$ per channel, respectively) over 512 channels for the joint EVN and the MERLIN only data, respectively. Only the MERLIN portion of this data set has so far been reduced and is presented here. The second data set (run2, December 11th-12th 2004) was a 13 hour MERLIN only experiment, including 5 antennas: CM, KN, DA, the 25-m Jodrell Bank Mk2 antenna (JB) and Tabley (TA). The spectral resolution was $0.976 \mathrm{kHz} / \mathrm{chan}$ ( $0.044 \mathrm{~km} \mathrm{~s}^{-1}$ per channel) over 512 channels. In both runs the telescopes were pointed, and the data correlated, at the position of the brightest methanol maser feature in IRS 1 , feature $A$ in Fig. 2 (see also Table 1).

First order calibration and translation into fits format was performed with tdproc at Jodrell Bank. Corrections to the primary amplitude calibration, phase calibration (self calibration

\footnotetext{
The EVN is a joint facility of European, Chinese, South African and others radio astronomy institutes.

2 The Multi-Element Radio Linked Interferometer Network is a National Facility run by the University of Manchester, UK.
} 
Table 1. Position, line of sight (LOS) velocity $(v)$ and offset $(r)$ from feature $A$ of the two maser components $F$ and $G$. The uncertainties in the positions and in the velocity widths are 50 mas $(135 \mathrm{AU})$ and $0.044 \mathrm{~km} \mathrm{~s}^{-1}$ respectively.

\begin{tabular}{ccccc}
\hline \hline Masers & \multicolumn{1}{c}{$\begin{array}{c}\mathrm{RA} \\
\mathrm{J} 2000\end{array}$} & $\begin{array}{c}\text { Dec } \\
\mathrm{J} 2000\end{array}$ & $\begin{array}{c}v / F W H M \\
\mathrm{~km} \mathrm{~s}^{-1}\end{array}$ & $\begin{array}{c}r \\
\operatorname{arcmin} / \mathrm{pc}\end{array}$ \\
\hline $\mathrm{A}$ & 231345.363 & 612810.55 & $-56.2 / 1.0$ & \\
$\mathrm{~F}$ & 231344.86 & 612648.1 & $-53.2 / 0.5$ & $1.36 / 1.06$ \\
$\mathrm{G}$ & 231401.78 & 612719.7 & $-48.8 / 0.9$ & $2.14 / 1.67$ \\
\hline
\end{tabular}

on the brightest emission channel) and image production were performed in AIPS. To locate all the maser emission, large $10 \times 10$ arcsec spectral cubes were made spanning the full observed velocity range.

In the cross correlation data from run1 (confirmed then by the analysis of the data from run2) emission was detected at the velocities of the known spectral features but also unexpectedly in some other spectral channels. The latter was large non noiselike side-lobe emission, indicating that maser emission at these velocities came from sources located outside of the map region. Maps were then iteratively re-centered until the new features, were brought close to the image centre.

The large position offsets of the new features from the correlated position cause large variations of the visibility phase over the integration time, and hence introduce amplitude errors on the visibilities. These do not affect feature position determination, but prevent us from making reliable images. Features $F$ $\& \mathrm{G}$ showed maximal phase changes of 0.6 and $1.8^{\mathrm{rad}}$ over the integration time of $7 \mathrm{~s}$, producing mean amplitude losses of $\sim 1$ and $8 \%$ respectively, on the baseline in Fig. 2 .

The positions of the masers were compared to those of $1.2 \mathrm{~mm}$ dust peak emission that were obtained in a MAMBO2/IRAM-30m map. The image was obtained in January 2004 using the MAMBO-2 bolometer array (Kreysa et al. 1998) installed at the IRAM 30 m telescope in the on-the-fly mode. The resulting angular resolution is $H P B W \sim 11^{\prime \prime}$ and the absolute pointing is accurate to within $\sim 3^{\prime \prime}$.

\section{Results}

\section{1. $\mathrm{CH}_{3} \mathrm{OH}$ masers features $\mathrm{F} \& \mathrm{G}$}

In the following, we denote the two new spectral features found during data reduction as $F$ and $G^{3}$. Their positions and other properties are summarised in Table 1.

The comparison of the cross correlated spectrum on the shortest baseline with the integrated spectrum of the maser emission shows discrepancy in the peak flux of feature $F \& G$ of $\sim 20 \%$ (Fig. 2). Unfortunately, the amplitude errors mentioned in Sect. 2 and the unreliable baseline subtraction in the integrated spectrum introduce errors in the amplitude that can be estimated to $15 \%$. These prevent us to invoque resolution as responsible for the missing flux. Our best estimate for the size of $F \& G$ (upper limit) is about half the fringe spacing of the shortest baseline, i.e. $\sim 85$ mas, or $260 \mathrm{AU}$, which is to be taken

${ }^{3}$ The nomenclature of all features follows the one adopted in Minier et al. (2000), based on the spatial distribution of the masers. as an upper limit. Note that in the higher spectral resolution data of run2 (not shown here), F \& G show clear multiplicity (at least two and three components for F \& $G$ respectively). Observations pointed at the new masers are necessary to obtain reliable and accurate maps of all features.

A review of archival EVN VLBI autocorrelation data for all antennas except Effelsberg shows that both $F$ and $G$ are clearly present in all experiments since 1997. These features always have similar flux ratios to spectral feature $A$ (within a variation of the latter of some $10 \%$ ). This indicates that the emission from $F$ and $G$ is probably non-variable. The new features are not detected in the autocorrelations from the Effelsberg $100 \mathrm{~m}$ antenna because they fall outside the HPBW of the primary beam at $5 \mathrm{~cm}$ (i.e. 1.6 arcmin). Note that despite the reduction in amplitude due to the primary beam attenuation a hint of feature $\mathrm{G}$ was present in the discovery spectrum for NGC 7538 taken with the 140 foot Green Bank telescope (Menten 1991).

\section{2. $\mathrm{CH}_{3} \mathrm{OH}$ masers in NGC 7538 IRS 9 \& $S$}

Figure 1 shows a general view of the NGC 7538 region. The $\mathrm{CH}_{3} \mathrm{OH}$ masers are clearly associated with the most embedded sites of high-mass star formation: IRS 1, IRS 9 and S. Masers in IRS 1 coincide with the $1.2 \mathrm{~mm}$ continuum peak emission (within the position accuracy) tracing cold dust emission from massive and deeply embedded protoclusters.

Maser feature $F$ coincides with the $1.2 \mathrm{~mm}$ dust continuum emission peak within $\sim 2^{\prime \prime}$ (Fig. 1). This is less than the position accuracy of the $1.2 \mathrm{~mm}$ data $\left(\sim 3^{\prime \prime}\right)$, and therefore we can state that they are cospatial. The methanol maser also coincides with other signposts of high-mass star formation in NGC $7538 \mathrm{~S}$ as reported in the literature, within the position accuracies (Sandell \& Sievers 2004; Sandell et al. 2003; Kameya et al. 1990). In Sandell et al. (2003) this protocluster/cloud is stated to be one of the most massive of the region, with $1000 M_{\odot}$ estimated within a circle of $20^{\prime \prime}$ (or $50000 \mathrm{AU}$ ) in diameter. The authors of that paper also report of a large rotating torus of some $14000 \mathrm{AU}$ in radius. Single grey-body Spectral Energy Distribution (SED) fitting of the cold dust emission in the FIR/(sub)mm/mm emission with an aperture of $60^{\prime \prime}$ of this source gives a dust temperature of $\sim 35 \mathrm{~K}$ and an emissivity in$\operatorname{dex} \beta=1.6$. This source lies some $10^{\prime \prime}$ to the south of IRS 11 , where a CO outflow is inferred by the distribution of the magnetic field orientation (Kameya et al. 1991). On the basis of the above multiple evidence, we consider the position of the methanol maser to be the most reliable for the protostellar object in NGC $7538 \mathrm{~S}$.

Maser feature $G$ is coincident within 1 " with the peak of the $1.2 \mathrm{~mm}$ dust emission. The position of $\mathrm{G}$ also agrees, within the position inaccuracies, with the high resolution positions of the peak emission of the continuum at 4.8 to $107 \mathrm{GHz}$ (van der Tak et al. 2000; van der Tak \& Menten 2005). Furthermore, one water vapour maser spot as well as one class I methanol maser spot seem also to coincide, within the positional accuracies, with feature G (Kameya et al. 1990; Sandell et al. 2005), although with an offset in velocity of some $6 \mathrm{~km} \mathrm{~s}^{-1}$. Because of the fact that class I methanol masers are mainly found in 
outflows (Kurtz et al. 2004), we argue that the newly detected maser $\mathrm{G}$ marks the driving source of the outflow (together with the continuum), while the other masers arise in the outflow shocks. This is supported by the geometry proposed in Sandell et al. (2005) for that source: we might observe the outflow from IRS 9 pole-on. The protostellar object in IRS 9 is accurately located both with the class II methanol maser and the $\mathrm{cm} / \mathrm{mm}$ continuum.

\section{Discussion}

The presence of three methanol maser sources associated to three different objects in the same star formation region offers a unique opportunity to study the evolutionary details of methanol maser bearing sources.

The methanol maser phase is considered to be relatively short as compared to the lifetime of the massive protostar, as short as a few $10^{4} \mathrm{yr}$ (van der Walt 2005). This implies that the sources hosting methanol masers at $6.7 \mathrm{GHz}$ should show very similar properties. The methanol masers in NGC 7538 (features $A, G \& F)$ on the other hand seem to mark massive young stellar objects at different stages of evolution: an IR-quiet protostellar object (NGC $7538 \mathrm{~S}$ ), an IR-bright protostellar object (IRS 9) and an UCH II region (IRS 1). The same distinction seems to be shown by the flux densities of the masers: $300,15,5 \mathrm{Jy}$ for $A, G, F$ respectively.

We propose 2 scenarios for this apparent sequence. The first and most intuitive is the evolutionary sequence. Feature $F$ might mark the youngest object in the region, feature $A$ the most evolved but still within the protostellar phase. This scenario is also supported by the presence of discs/outflows in the maser sources: in F a large scale rotating torus is detected but no outflow (Sandell \& Sievers 2004); in G a powerful, probably very young, outflow is mapped (Sandell et al. 2005); in A a clear disc/outflow geometry is visible (e.g. Gaume et al. 1995; Pestalozzi et al. 2004; De Buizer \& Minier 2005). More evidence has to be collected on the capacity of methanol masers to provide such accuracy in the age the sources hosting them.

The second scenario invokes geometry. The difference in $1.2 \mathrm{~mm}$ dust emission peak flux among the methanol maser bearing sources could be due to different viewing angles to the source. IRS 1 is seen edge-on, showing the thick dust in its disc (Gaume et al. 1995; Pestalozzi et al. 2004). IRS 9 is suggested to be seen close to pole-on (Sandell et al. 2005): the disc would be seen almost face-on, not contributing significantly to the $1.2 \mathrm{~mm}$ flux. NGC $7538 \mathrm{~S}$ is still deeply embedded, so dust is close to uniformly distributed around the source. This interpretation seems to be more difficult to accept, because e.g. IRS 1 suffers of significant contamination by the very close IRS 2 and IRS 3.

\section{Summary and conclusions}

From the high spatial resolution MERLIN observations of 6.7 GHz methanol maser emission toward the massive star forming region NGC 7538 we have detected and positioned two new spectral features. We note that this achievement was possible only thanks to the intermediate resolution of the MERLIN with its shortest baselines. We conclude the following:

- on the basis of the accepted idea that methanol masers are the most efficient tracers of young and embedded massive protostars, the accurate location (to within 50 mas or $135 \mathrm{AU}$ ) of $\mathrm{F}$ and $\mathrm{G}$ is equivalent with the accurate location of the massive protostars in NGC 7538 IRS 9 and NGC $7538 \mathrm{~S}$;

- the variety of objects marked by methanol maser emission could be due to different ages or viewing angle geometry. To test these two scenarios, more accurate observations (targeted toward F \& G) are required.

Acknowledgements. M.P. thanks Tom Muxlow for his help during the data calibration at the Jodrell Bank Observatory. We thank all the participants of the NGC 7538 collaboration for the very fruitful discussions that contributed to the acheivement of this paper.

\section{References}

Davis, C., Moriartry-Schieven, G., Eislöffel, J., Hoare, M., \& Ray, T. 1998, ApJ, 115, 1118

De Buizer, J., \& Minier, V. 2005, ApJ, 628, L151

Gaume, R., Goss, W., Dickel, H., Wilson, T., \& Johnston, K. 1995, ApJ, 438, 776

Hill, T., Burton, M., Minier, V., et al. 2005, MNRAS, 363

Kameya, O., K.-I., M., Kawabe, R., \& Ishiguro, M. 1990, ApJ, 355, 562

Kameya, O., Hirano, N., Kawabe, R., \& Campbell, B. 1991, in Proceedings of a Conference at the University of Manchester, March 26-30, 1990, Cambridge, ed. R. James, \& T. Millar (Cambridge University Press), 111

Kreysa, E., Gemünd, H., Gromke, J., et al. 1998, SPIE, 3357, 319

Kurtz, S., Hofner, P., \& Vargas Alvarez, C. 2004, ApJS, 155, 149

Menten, K. 1991, ApJ, 380, L75

Minier, V., Booth, R., \& Conway, J. 1998, A\&A, 336, L5

Minier, V., Booth, R., \& Conway, J. 2000, A\&A, 362, 1093

Minier, V., Conway, J., \& Booth, R. 2001, A\&A, 369, 278

Minier, V., Burton, M., Hill, T., et al. 2005, A\&A, 429, 945

Motte, F., Schilke, P., \& Lis, D. 2003, ApJ, 582, 277

Ojha, D., Tamura, M., Nakajima, Y., et al. 2004, ApJ, 616, 1042

Pestalozzi, M., Elitzur, M., Conway, J., \& Booth, R. 2004, ApJ, 603, L113

Purcell, C., Balasubramanyam, R., Burton, M., et al. 2005, MNRAS, in press

Sandell, G., \& Sievers, A. 2004, ApJ, 600, 269

Sandell, G., Wright, M., \& Forster, J. 2003, ApJ, 590, L45

Sandell, G., Goss, W., \& Wright, M. 2005, ApJ, 621, 839

Sobolev, A., Cragg, D., \& Godfrey, P. 1997, MNRAS, 288, L39

van der Walt, D. 2005, MNRAS, 360, 153

van der Tak, F., van Dishoeck, E., \& Caselli, P. 2000, A\&A, 361, 327

van der Tak, F., \& Menten, K. 2005, A\&A, 437, 947

Walsh, A., Burton, M., Hylard, A., \& Robinson, G. 1998, MNRAS, 301,640 